\title{
Cinética de secagem do resíduo de abacaxi enriquecido
}

\author{
Hofsky V. Alexandre1, Flávio L. H. da Silva² ${ }^{\text {, Josivanda P. Gomes }}{ }^{3}$, \\ Osvaldo S. da Silva ${ }^{4}$, João P. D. Carvalho ${ }^{5}$ \& Ezenildo E. de Lima ${ }^{6}$
}

\begin{abstract}
RESUMO
O Brasil é um dos maiores produtores de abacaxi do mundo e o maior da América do Sul. O fruto é a parte comercializável da planta enquanto o restante é formado por caule, folha, casca, coroa e talos, considerado resíduo agrícola e não tem sido devidamente aproveitado, resultando em perdas econômicas. Caracterizar e avaliar a cinética de secagem do resíduo (casca) de abacaxi (Ananas comosus L.) enriquecido utilizando a levedura Saccharomyces cerevisiae em leito estático, em camada fina de secagem, utilizando-se de planejamento experimental fatorial, foi o objetivo deste estudo. A temperatura $\left(40\right.$ a $\left.60^{\circ} \mathrm{C}\right)$ e a velocidade do ar de secagem $\left(0,8\right.$ a $\left.1,3 \mathrm{~m} \mathrm{~s}^{-1}\right)$ são as variáveis do processo. $\mathrm{O}$ resíduo enriquecido e caracterizado apresentou alto teor de matéria seca $(90,27 \%)$ e elevado teor de proteína $(20,21 \%)$ em relação ao resíduo in natura $(7,61 \%)$. A cinética de secagem ocorreu no período de taxa decrescente. Os modelos Page, Henderson \& Pabis e Lewis, ajustaram os dados experimentais satisfatoriamente, sendo Page discretamente superior aos demais. Verificou-se influência da variável temperatura apenas para a variável resposta umidade, não sendo observada influência estatisticamente significativa da velocidade do ar de secagem.
\end{abstract}

Palavras-chave: Ananas comosus L., Saccharomyces cerevisiae, secagem

\section{Drying kinetics of enriched pineapple residue}

\begin{abstract}
Brazil is the largest producer of pineapple (Ananas comosus L.) in South America and one of the largest in the world. The fruit is the marketable part of the plant, while the rest formed by stem, leaves, peel, crowns and shafts is considered as agricultural residue and is not properly used, resulting in economic loss. The aim of this study was to characterize and to evaluate the drying kinetics of enriched pineapple residue (peel) using Saccharomyces cerevisiae yeast in static bed in fine layer, using of experimental factorial planning. The temperature $\left(40\right.$ to $\left.60{ }^{\circ} \mathrm{C}\right)$ and the wind speed $\left(0.8\right.$ to $\left.1.3 \mathrm{~m} \mathrm{~s}^{-1}\right)$ were the process variables. The characterized enriched residue exhibited a high dry matter content $(90.27 \%)$ and a high protein content $(20.21 \%)$ in relation to fresh residue $(7.61 \%)$. The drying kinetics occurred in falling rate period. The Page, Henderson \& Lewis and Pabis models adjusted satisfactorily to experimental data, being the Page model slightly superior to the others. Influence of the temperature variable was verified only for the moisture response variable, while no significant influence of the wind speed of drying was observed.
\end{abstract}

Key words: Ananas comosus L., Saccharomyces cerevisiae, drying

\footnotetext{
1 PNPD/CAPES/UNIT, Av. Murilo Dantas, 300, Bairro Farolândia, CEP 49032-490, Aracaju, SE. Fone: (79) 32182190. E-mail: hofsky@gmail.com ${ }^{2}$ CT/UFPB, Campus I, Cidade Universitária, CEP 58051-900, João Pessoa, PB. Fone: (83) 3216-7357. E-mail: flavioluizh@yahoo.com.br

${ }^{3}$ UAEA/UFCG, Campus I, Av. Aprígio Veloso, 882, Bairro Universitário, CEP 58429-140, Campina Grande, PB. Fone: (83) 2101-1055, E-mail: josi@deag.ufcg.edu.br

${ }^{4}$ CCTA/UFCG, Campus Pombal, R. Jairo Vieira Feitosa, 1770, Bairro dos Pereiros, CEP 58840-000, Pombal, PB. Fone: (83) 3431-4006. E-mail: osvaldo@ccta.ufcg.edu.br

${ }^{5}$ Graduando em Engenharia Química - CCT/UFCG, Campus I, Av. Aprígio Veloso, 882, Bairro Universitário, CEP 58429-140, Campina Grande, PB. Fone: (83) 2101-1113. E-mail: jpd_carvalho@hotmail.com

${ }^{6}$ CTPE/IFPE, Campus Afogados da Ingazeira, Rua Edson Barbosa de Araújo, S/N, Bairro Manoela Valadares, CEP 56800-000, Afogados da Ingazeira, PE. Fone: (81) 2125-1630, ezenildo.lima@afogados.ifpe.edu.br
} 


\section{INTRODUÇÃO}

O Brasil é um dos maiores produtores de abacaxi ressaltandose, como os principais, os estados são da Paraíba, Pará e Minas Gerais. Brito Neto et al. (2008) afirmam que as condições brasileiras para a produção deste fruto visando aos mercados interno e externo, lhes asseguram vantagens comparativas em relação aos países concorrentes devido ao clima favorável, grande disponibilidade de área e de tecnologias.

A cultivar 'Pérola' é muito apreciada no mercado interno graças à sua polpa suculenta e saborosa, considerada insuperável para o consumo ao natural fazendo com que os frutos tenham grande potencial de comercialização internacional pois também são muito apreciados no Mercosul e na Europa (Souto et al., 2004). Este fruto tropical é muito utilizado como matéria-prima para a fabricação de diversos produtos alimentícios (Costa et al., 2007).

A crescente preocupação com o meio ambiente incentiva a viabilização de projetos que levam à sustentabilidade do sistema de produção industrial. A indústria de alimentos produz uma série de resíduos com alta capacidade de reutilização. Com isto, minimiza-se o impacto ambiental desses tipos de indústria na região em que estão situadas e ainda se agrega valor aos produtos do mercado (Pelizer et al., 2007).

O resíduo da industrialização do abacaxi, como qualquer outro subproduto quando fornecido de forma exclusiva, não atende às necessidades de manutenção dos animais (Ribeiro et al., 1993) uma vez que apresenta baixo teor de proteína e elevado percentual de fibra em detergente neutro (FDN). Essas restrições, de acordo com Martin (1997), resultam em baixos coeficientes de digestibilidade e, consequentemente, em baixo consumo voluntário.

Para utilização do resíduo agroindustrial do abacaxi na alimentação animal torna-se conveniente estabelecer um equilíbrio entre os nutrientes da dieta para que haja fermentação eficiente no rúmen objetivando otimizar o crescimento microbiano e maximizar a digestão da fibra, atendendo às necessidades nutricionais dos ruminantes (Correia et al., 2006).

Uma das maiores dificuldades para se empreender uma ação concreta de beneficiamento e destinação de resíduos, além de problemas econômicos, políticos e administrativos, está na ausência de técnicas de processamento e beneficiamento desses resíduos (Della et al., 2005).

$\mathrm{O}$ processo de enriquecimento proteico em rejeitos agroindustriais utilizando-se micro-organismo pode ser realizado através de uma fermentação (cultivo) semissólida que possibilite a utilização desses rejeitos como substrato (Campos et al., 2005). Porém se deve criar, após o enriquecimento, condições nas quais o resíduo enriquecido esteja protegido e livre das ações maléficas dos fatores que condicionam sua deterioração.

A tecnologia de alimentos tem, entre seus elementos de estudo, o aumento da vida útil do produto alimentício convertendo-os em produtos mais estáveis que possam ser estocados por longos períodos, tendo como técnicas mais importantes o congelamento e a secagem.

A secagem é o processo comercial mais utilizado para preservar o alimento e, comparado com outros métodos preservativos para períodos longos, como a centrifugação, o enlatamento, os tratamentos químicos e a irradiação, entre outros, é de baixo custo e de operação mais simples. De acordo com Ferreira \& Pena (2010) trata-se de um fenômeno complexo que envolve simultaneamente a transferência de calor e massa podendo abranger ainda a transferência de quantidade de movimento.

Esta é a operação unitária mais empregada na conservação de alimentos; ela tem, como objetivo, reduzir o teor de água do produto possibilitando o aumento de sua vida-de-prateleira (redução da atividade de água). Além de que a redução do volume do produto facilita seu transporte e armazenamento (Ribas et al., 2000).

As informações contidas nas curvas de secagem são de fundamental importância para o desenvolvimento de processos e para o dimensionamento de equipamentos; com elas, pode-se estimar o tempo de secagem de certa quantidade de produtos e, com o tempo necessário para a produção, estima-se o gasto energético que refletirá no custo de processamento e influenciará no preço final do produto (Vilela \& Artur, 2008).

As curvas de secagem em camada delgada variam com a espécie, variedade, condições ambientais e métodos de preparo pós-colheita, entre outros fatores; neste sentido, diversos modelos matemáticos têm sido utilizados para descrever o processo de secagem de produtos agrícolas cuja aplicabilidade na predição de dados experimentais alcança grande importância na prática haja vista que permite a redução dos exaustivos ensaios de laboratório. É considerável o número de modelos matemáticos, teóricos, semiempíricos e empíricos na literatura, que podem ser empregados na predição de dados de secagem em camada delgada (Akpinar et al., 2003; Mohapatra \& Rao, 2005; Resende et al., 2008).

Conduziu-se este trabalho com o objetivo de verificar os efeitos das variáveis (temperatura e velocidade do ar de secagem) para o resíduo (casca) enriquecido com a levedura Saccharomyces cerevisiae, sobre a cinética de secagem em camada fina em secador convectivo e ajustá-los aos modelos descritos na literatura.

\section{Material e Métodos}

Este trabalho foi realizado no Laboratório de Engenharia Bioquímica em conjunto com o Laboratório de Transferência em Meios Porosos e Sistemas Particulados, ambos pertencentes à Unidade Acadêmica de Engenharia Química, no Centro de Ciências e Tecnologia da Universidade Federal de Campina Grande, PB.

A matéria-prima utilizada no estudo foi a casca do abacaxi pérola (Ananas comosus L.); o fruto foi adquirido na EMPASA na cidade de Campina Grande, PB. No laboratório o resíduo (casca) foi separado da polpa em seguida acondicionado em sacos de polietileno e só então armazenado em freezer a $-22^{\circ} \mathrm{C}$ até o momento de ser utilizado nos experimentos.

Para o enriquecimento proteico do substrato em estudo foram utilizados: levedura Saccharomyces cerevisiae, prensada, do tipo comercial, fermento biológico fresco para a fabricação de pães e seus derivados da marca Levapan, com $70 \%$ de umidade e $45 \%$ de proteína bruta (base seca). Utilizaram-se 
também as condições maximizadas visando à fermentação semissólida obtidas por Oliveira (2007); este período de enriquecimento de $48 \mathrm{~h}$, a concentração de levedura $3 \%$ e a temperatura de $34{ }^{\circ} \mathrm{C}$.

Para sua caracterização, uma parcela do resíduo enriquecido foi submetida à secagem em estufa com circulação de ar, na temperatura de $55^{\circ} \mathrm{C}$, durante $72 \mathrm{~h}$; em seguida o material, já seco, foi triturado em moinho elétrico e colocado em recipientes de plástico herméticos e armazenados em temperatura ambiente $\left(\approx 25^{\circ} \mathrm{C}\right)$ para análise.

$\mathrm{O} \mathrm{pH}$ e a matéria seca foram determinados de acordo com a metodologia descrita em Brasil (2005). Para determinação da matéria seca a amostra foi colocada em estufa a $105^{\circ} \mathrm{C}$, até atingir peso constante. A determinação de cinzas foi realizada seguindo-se a metodologia descrita pela AOAC (1997). Com o teor de cinzas, determinou-se a matéria orgânica, em que $\mathrm{MO}$ $=100$ - Cinzas.

Para determinações de fibra detergente ácido (FDA) e fibra detergente neutro (FDN) a metodologia utilizada foi a técnica proposta por Silva (1998). O valor da hemicelulose foi determinado através da subtração do valor de fibra em detergente neutro (FDN) pelo de fibra em detergente ácido (FDA). Na determinação de açúcares redutores (ART, frutose, glicose e sacarose) utilizou-se a metodologia descrita por Miller (1959).

O teor de proteína bruta foi determinado pelo método semimicro Kjeldahl com adaptação para nitrogênio $(\mathrm{N})$, por espectrofotometria UV-VIS, segundo o método descrito em Le Poidevin \& Robinson (1964). Para calcular a porcentagem de proteína da amostra a partir da quantidade de nitrogênio orgânico existente foi preciso considerar que, em média, as proteínas possuem $16 \%$ de nitrogênio e que são totalmente digeridas fazendo com que o fator de conversão de porcentagem de nitrogênio em porcentagem de proteína seja 6,25 , ou seja, $\mathrm{PB}(\%)=\mathrm{N}(\%) \times 6,25$.

Para realização deste trabalho foram definidas, como variáveis de entrada (variáveis independentes) para verificação dos seus efeitos sobre a cinética de secagem, a temperatura (40, 50 e $\left.60^{\circ} \mathrm{C}\right)$ e a velocidade do ar de secagem $(0,8,1,3$ e $1,8 \mathrm{~m}$ $\left.\mathrm{s}^{-1}\right)$. As variáveis dependentes, respostas, em estudo, foram a proteína na umidade de equilíbrio e a umidade no tempo 120 $\min$.

A Tabela 1 apresenta a matriz de planejamento fatorial $2^{2}$, totalizando em 7 experimentos, dentre os quais 4 ensaios distintos e mais 3 no ponto central, que são 3 ensaios idênticos com uma das finalidades de verificar a reprodução dos ensaios (verificação dos erros).

Tabela 1. Matriz de planejamento fatorial completo $2^{2}$

\begin{tabular}{ccccc}
\hline \multirow{2}{*}{ Ensaios } & \multicolumn{2}{c}{ Temperatura } & \multicolumn{2}{c}{ Velocidade do ar } \\
\cline { 2 - 5 } & Codificada & Real $\left({ }^{\mathbf{C}} \mathbf{C}\right)$ & Codificada & Real $\left(\mathbf{m ~ s}^{\mathbf{- 1}}\right)$ \\
1 & -1 & 40 & -1 & 0,8 \\
2 & +1 & 60 & -1 & 0,8 \\
3 & -1 & 40 & +1 & 1,8 \\
4 & +1 & 60 & +1 & 1,8 \\
5 & 0 & 50 & 0 & 1,3 \\
6 & 0 & 50 & 0 & 1,3 \\
7 & 0 & 50 & 0 & 1,3 \\
\hline
\end{tabular}

Para os experimentos de secagem utilizou-se um secador convectivo (Figura 1). A quantidade de água evaporada durante o processo foi obtida por pesagens descontínuas das amostras e o esquema de pesagem seguiu intervalos de 5,10 , e $30 \mathrm{~min}$, até o final do processo. Os testes foram realizados até que as variações do peso das amostras fossem insignificantes; em seguida, as amostras foram colocadas em estufa, na uma temperatura de $105^{\circ} \mathrm{C}$, por $24 \mathrm{~h}$, para determinação da matéria seca.

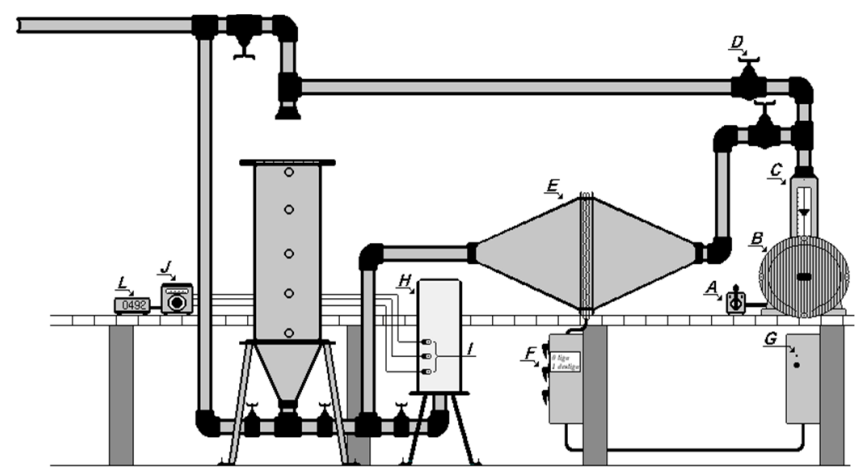

Legenda:

A. Chave do compressor; B. Ventilador radial; C. Medidor de vazão; D. Válvula reguladora E. Aquecedor elétrico; F. Chave das resistências; G. Chave do sistema de controle; H. Câmara de secagem; I. Termopares; J. Controlador de temperatura; L. Milivoltímetro digital

Figura 1. Esquema do equipamento utilizado na rea-

lização da secagem convectiva

Inicialmente, foram determinadas as condições do ambiente como temperatura e umidade relativa do ar utilizando-se um higrômetro; verificou-se a velocidade do ar de secagem mediante um anemômetro de ventoinha; a secagem foi realizada em camada delgada de aproximadamente $12 \mathrm{~mm}$ de espessura; as amostras do resíduo enriquecido com uma massa média de $115 \mathrm{~g}$ foram colocadas em uma cesta de material metálico, em tela fina de aço inox.

Com os dados das pesagens das amostras durante a secagem calcularam-se as razões de umidade ou umidade adimensional (Eq. 1).

$$
\mathrm{RX}=\frac{\mathrm{X}-\mathrm{Xe}}{\mathrm{Xo}-\mathrm{Xe}}
$$

em que:

RX - razão de umidade ou umidade adimensional

$\mathrm{X}$ - umidade absoluta, bs

Xo - umidade inicial, bs

Xe - umidade de equilíbrio, bs

Para o ajuste matemático das curvas de secagem dos resíduos foram utilizadas as equações de Lewis, Page e Henderson \& Pabis (Eqs. 2, 3 e 4) ajustadas com o auxílio do processador matemático Statistica, versão 5.0.

$$
\begin{gathered}
\mathrm{RX}=\exp ^{(-\mathrm{kt})} \\
\mathrm{RX}=\mathrm{e}^{-k \mathrm{tt}^{\mathrm{n}}} \\
\mathrm{RX}=\mathrm{a} \exp (-\mathrm{kt})
\end{gathered}
$$


em que:

$$
\begin{array}{ll}
\mathrm{RX} & \text { - razão de umidade, adimensional } \\
\mathrm{t} & \text { - tempo de secagem, } \mathrm{s} \\
\mathrm{k} & \text { - constante de secagem, } \mathrm{s}^{-1} \\
\mathrm{a}, \mathrm{n} & \text { - constante do modelo, adimensional. }
\end{array}
$$

\section{Resultados E Discussẽo}

Os resultados obtidos para a caracterização físico-química da casca do abacaxi enriquecido estão na Tabela 2; por sua vez, o teor de matéria seca presente no resíduo de abacaxi enriquecido apresenta valor médio de 90,27\%. Segundo as normas de alimentação propostas pela NRC (1989) recomenda-se que o teor de matéria seca contida na alimentação do rebanho bovino apresente um percentual em torno de 16,5\%; em termos de matéria seca o resíduo de abacaxi enriquecido apresenta uma quantidade suficiente para suprir a carência deste parâmetro na alimentação animal.

Tabela 2. Caracterização físico-química do resíduo do abacaxi enriquecido

\begin{tabular}{lc}
\hline \multicolumn{1}{c}{ Parâmetros } & Casca \\
Matéria seca (\%) & $90,27 \pm 1,16 \%$ \\
pH & $5,84 \pm 0,01$ \\
Cinzas (\%) & $6,50 \pm 0,13 \%$ \\
Matéria orgânica (\%) & $93,50 \pm 0,13 \%$ \\
ART (\%) & $0,49 \pm 0,01$ \\
FDN (\%) & $57,63 \pm 0,33$ \\
FDA (\%) & $22,04 \pm 0,64$ \\
Hemicelulose & $35,59 \pm 0,30$ \\
Proteína bruta (\%) & $20,21 \pm 0,63$ \\
\hline
\end{tabular}

O teor de cinzas e de matéria orgânica apresentado neste trabalho foi maior quando comparado com os valores reportados por Costa et al. (2007) que também fizeram a caracterização física de pós alimentícios obtidos de resíduos de casca do abacaxi proveniente da industrialização deste fruto.

$\mathrm{O}$ teor de açúcares encontrado no resíduo in natura foi de 3,64\%; para o enriquecimento proteico em meio sólido o micro-organismo utilizado foi a Saccharomyces cerevisiae que metaboliza carboidratos solúveis podendo-se afirmar, então, que este material tem potencial para o processo de enriquecimento proteico. A quantidade de açúcares totais encontrada na casca enriquecida foi de $0,49 \%$, com o que se pode considerar que $86,5 \%$ do açúcar contido na casca in natura foram metabolizados pela levedura.

Os valores de FDN e FDA encontrados neste trabalho para a casca de abacaxi enriquecido foram de 57,63 e 22,04\%, respectivamente. Segundo Figueiredo (1996) os alimentos com percentuais de FDN acima de 35\% garantem teor normal de gordura do leite e, segundo as recomendações do NRC (1989) para alimentação de vacas em lactação o valor exigido é no mínimo de $21 \%$ de FDA; o teor de hemicelulose para a casca foi de $35,59 \%$.

O teor de proteína médio foi de $20,21 \%$ dentro, portanto, das normas definidas pela NRC (1989) para vacas em lactação que precisam receber teor de proteína diária entre 20 a $24 \%$. Os resultados dos experimentos realizados para o enriquecimento proteico do resíduo do abacaxi se encontram na Tabela 3.
Tabela 3. Dados do enriquecimento proteico do resíduo do abacaxi

\begin{tabular}{cccc}
\hline \multirow{2}{*}{ Resíduos } & PBin & PBe & AP \\
\cline { 2 - 4 } Casca & \multicolumn{1}{c}{$(\%)$} & \\
\hline
\end{tabular}

Pbin - Proteína bruta in natura do resíduo; Pbe - Proteína bruta do enriquecido; AP - Aumento proteico

Mediante os dados da Tabela 3 verifica-se que, após o enriquecimento proteico o substrato aumentou expressivamente seu teor de proteína para $165,57 \%$, o qual é superior ao valor encontrado por Oliveira (2007) durante o enriquecimento proteico de resíduos agroindustriais para utilização na alimentação animal.

Na Figura 2 estão representadas as cinéticas de secagem da casca de abacaxi enriquecida nas temperaturas e velocidade definidas no planejamento fatorial completo $2^{2}$ (Tabela 1) na forma do adimensional de umidade em função do tempo.

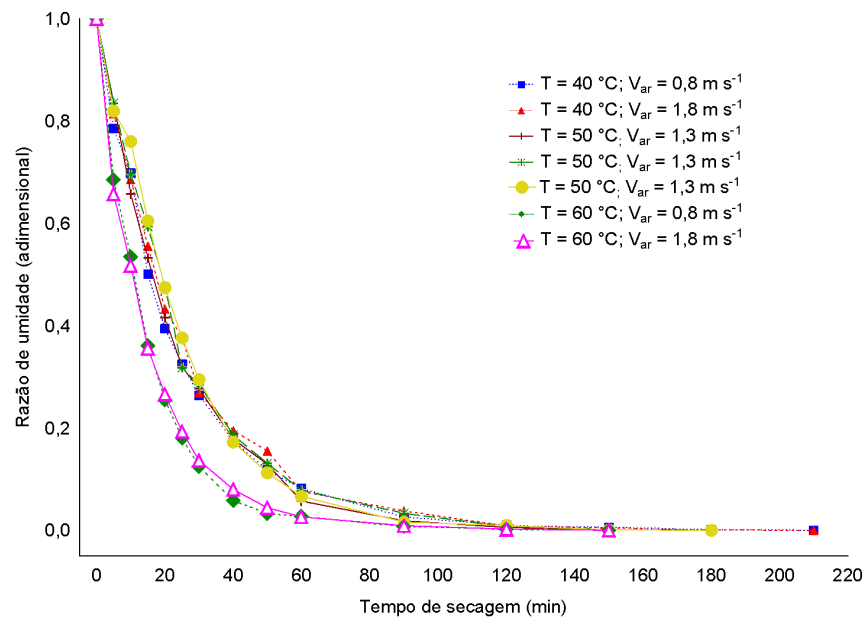

Figura 2. Curvas de secagem da casca de abacaxi enriquecida nas temperaturas e velocidades de secagem estudadas

Verifica-se que a temperatura é a variável de maior influência no processo visto que, para mesmas temperaturas, porém com velocidades diferentes, o tempo de secagem é o mesmo; Fiorentin et al. (2010) observaram comportamento semelhante ao avaliar as características cinéticas envolvidas no processo de secagem do bagaço de laranja.

Utilizando o planejamento experimental na avaliação da cinética de secagem de caju para identificação dos efeitos da temperatura e velocidade do ar de secagem, Gouveia et al. (2002) verificaram que a temperatura foi o fator que exerceu maior influência no processo.

Babalis et al. (2006) observaram o efeito da temperatura na secagem de figos nas temperaturas de $55-85^{\circ} \mathrm{C}$ e velocidade do ar de 0,5-3,0 $\mathrm{m} \mathrm{s}^{-1}$ e concluíram que o aumento da taxa de secagem com o aumento da temperatura é uma tendência amplamente verificada para a maioria dos produtos.

Carlesso et al. (2005) realizaram um experimento com secagem de sementes de maracujá em camada delgada utilizando um protótipo de secador de leito fixo, com três níveis de temperatura do ar de secagem $\left(30,37\right.$ e $\left.40^{\circ} \mathrm{C}\right)$ e três níveis de fluxo de ar para secagem $\left(24,48\right.$ e $\left.71 \mathrm{~m}^{3} \mathrm{~min}^{-1} \mathrm{~m}^{2}\right) \mathrm{e}$ 
constataram que o tempo de secagem diminuiu em função do aumento da temperatura porém não houve influência da vazão específica do ar de secagem.

Concluiu-se, então, que a velocidade do ar de secagem não apresentou efeito sobre as curvas de secagem nos níveis estudados, sugerindo que a resistência externa não influenciou as condições de operações utilizadas e que o controle da secagem dependeu da difusão interna do produto.

Na Tabela 4 se encontram os valores dos parâmetros dos modelos Page, Henderson \& Pabis e Lewis, ajustados aos dados experimentais das cinéticas de secagem da casca de abacaxi enriquecido nas temperaturas e velocidades estudadas, os coeficientes de determinação $\left(\mathrm{R}^{2}\right)$ e os desvios quadráticos médios (DQM).

Tabela 4. Parâmetros estimados pela equação de Page, Henderson \& Pabis e Lewis para os dados de secagem, coeficiente de determinação e desvios quadráticos médios (DQM) para casca de abacaxi enriquecida

\begin{tabular}{|c|c|c|c|c|c|c|c|}
\hline \multirow{2}{*}{ Modelo } & \multirow{2}{*}{ Ensaio } & \multirow{2}{*}{$\begin{array}{c}\mathrm{T} \\
\left({ }^{\circ} \mathrm{C}\right)\end{array}$} & \multirow{2}{*}{$\begin{array}{c}V_{\mathrm{ar}} \\
\left(\mathrm{m} \mathrm{s}^{-1}\right)\end{array}$} & \multicolumn{2}{|c|}{ Parâmetro } & \multirow{2}{*}{$\mathrm{R}^{2}$} & \multirow{2}{*}{ DQM } \\
\hline & & & & $k\left(s^{-1}\right)$ & $n$ & & \\
\hline \multirow{7}{*}{ Page } & 1 & 40 & 0,8 & 0,0436 & 1,0030 & 0,9985 & 0,017 \\
\hline & 2 & 60 & 0,8 & 0,0638 & 0,9934 & 0,9993 & 0,011 \\
\hline & 3 & 40 & 1,8 & 0,0389 & 1,0122 & 0,9992 & 0,013 \\
\hline & 4 & 60 & 1,8 & 0,0890 & 0,9011 & 0,9993 & 0,011 \\
\hline & 5 & 50 & 1,3 & 0,0376 & 1,0432 & 0,9995 & 0,010 \\
\hline & 6 & 50 & 1,3 & 0,0288 & 1,1031 & 0,9985 & 0,017 \\
\hline & 7 & 50 & 1,3 & 0,0204 & 1,1986 & 0,9986 & 0,017 \\
\hline \multirow{7}{*}{$\begin{array}{c}\text { Henderson } \\
\& \text { Pabis }\end{array}$} & 1 & 40 & 0,8 & 0,0441 & 1,0018 & 0,9985 & 0,017 \\
\hline & 2 & 60 & 0,8 & 0,0677 & 0,9951 & 0,9993 & 0,011 \\
\hline & 3 & 40 & 1,8 & 0,0405 & 1,0036 & 0,9992 & 0,013 \\
\hline & 4 & 60 & 1,8 & 0,0662 & 0,9769 & 0,9986 & 0,015 \\
\hline & 5 & 50 & 1,3 & 0,0438 & 1,0143 & 0,9994 & 0,011 \\
\hline & 6 & 50 & 1,3 & 0,0410 & 1,0225 & 0,9979 & 0,021 \\
\hline & 7 & 50 & 1,3 & 0,0440 & 1,0330 & 0,9964 & 0,028 \\
\hline \multirow{7}{*}{ Lewis } & 1 & 40 & 0,8 & \multicolumn{2}{|c|}{0,0439} & 0,9986 & 0,017 \\
\hline & 2 & 60 & 0,8 & \multicolumn{2}{|c|}{0,0681} & 0,9993 & 0,011 \\
\hline & 3 & 40 & 1,8 & \multicolumn{2}{|c|}{0,0403} & 0,9992 & 0,013 \\
\hline & 4 & 60 & 1,8 & \multicolumn{2}{|c|}{0,0678} & 0,9983 & 0,017 \\
\hline & 5 & 50 & 1,3 & \multicolumn{2}{|c|}{0,0430} & 0,9993 & 0,012 \\
\hline & 6 & 50 & 1,3 & \multicolumn{2}{|c|}{0,0399} & 0,9976 & 0,022 \\
\hline & 7 & 50 & 1,3 & \multicolumn{2}{|c|}{0,0386} & 0,9957 & 0,030 \\
\hline
\end{tabular}

Verificou-se que os modelos utilizados ajustaram satisfatoriamente os dados experimentais, com modelo de Page sinalizando discretamente maiores valores de $\mathrm{R}^{2} \mathrm{e}$ os menores valores de DQM para todas as temperaturas, ajustando-se melhor aos dados observados.

Azoubel et al. (2010) relataram, avaliando a cinética de secagem da banana CV Pacovan nas temperaturas de 50 e 70 ${ }^{\circ} \mathrm{C}$ e velocidade do ar de $3,0 \mathrm{~m} \mathrm{~s}^{-1}$, em secador de leito fixo, utilizando ultra-som como pré-tratamento, que o modelo de Page apresentou melhor ajuste aos dados experimentais.

Observou-se ainda, na Tabela 4, que todos os modelos mostraram valores de $\mathrm{R}^{2}$ superiores a 0,99 e valores de DQM inferiores a 0,03 podendo ser utilizados na estimativa das curvas de secagem da casca de abacaxi enriquecido.

Analisando-se o comportamento do modelo de Page constatou-se que o parâmetro $\mathrm{k}$, que representa a constante da taxa de secagem, aumentou com o aumento da temperatura quando se analisam as temperaturas de 40 e $60{ }^{\circ} \mathrm{C}$; entretanto, a temperatura de $50{ }^{\circ} \mathrm{C}$ apresentou, para $\mathrm{k}$, valores inferiores aos encontrados para a temperatura de 40 e $60{ }^{\circ} \mathrm{C}$ enquanto o parâmetro $\mathrm{n}$ diminuiu com o aumento da temperatura para as temperaturas de 40 e $60{ }^{\circ} \mathrm{C}$, e para a temperatura de $50{ }^{\circ} \mathrm{C}$ foram superiores aos valores para temperaturas de 40 e $60^{\circ} \mathrm{C}$.

Comportamento similar em relação ao parâmetro $\mathrm{k}$ quanto ao acréscimo da temperatura, foi reportado por Doymaz \& Pala (2003) estudando a secagem de milho nas temperaturas de 55 e $65{ }^{\circ} \mathrm{C}$. Concluindo-se que os valores dos parâmetros n para o modelo de Page e a para o modelo de Henderson \& Pabis, utilizados para ajuste dos dados da cinética de secagem da casca de abacaxi enriquecida, foram próximos da unidade, o que reduziu essas equações ao modelo de Lewis.

Na Figura 3 se encontram as curvas de secagem da casca de abacaxi enriquecida, nas temperaturas estudadas, com ajustes pelo modelo de Page.

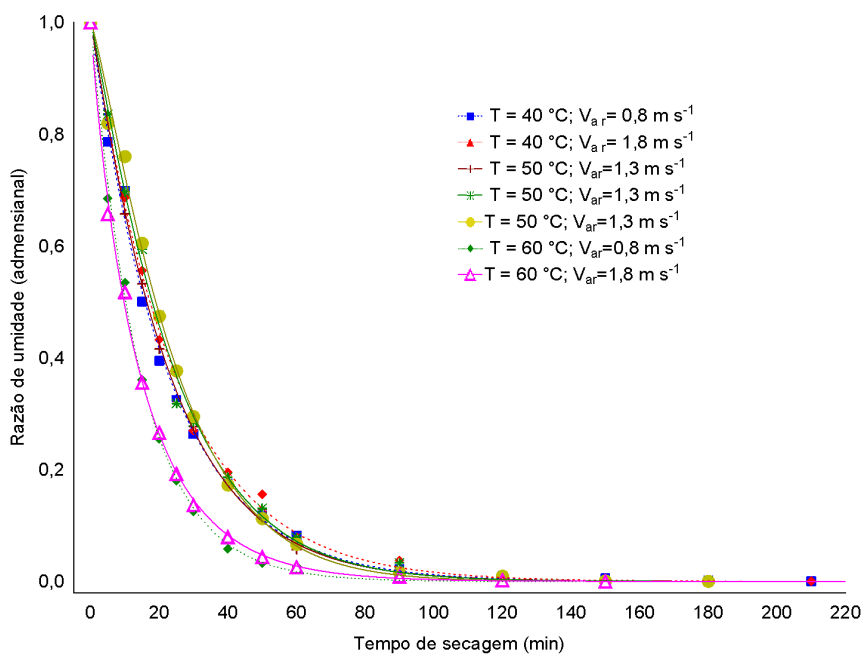

Figura 3. Curvas de secagem da casca de abacaxi enriquecida para as temperaturas estudadas com ajuste pelo modelo de Page

Sousa et al. (2006) relataram que o modelo de Page apresentou melhor ajuste aos dados experimentais da secagem do farelo de mamona (Ricinus communis $\mathrm{L}$.), com temperaturas de $50,60,70$ e $80^{\circ} \mathrm{C}$ e espessuras de camada de 1,3 e $5 \mathrm{~cm}$.

A análise dos resultados obtidos para umidade (120 min) da casca de abacaxi enriquecida, foi realizada através de métodos estatísticos de acordo com o planejamento fatorial simples $2^{2}$ com três pontos centrais cuja matriz estatística está resumida na Tabela 5. As variáveis reais e a variável resposta para os ensaios de umidade $\left(t_{120}\right)$ e teor de proteína enriquecido seco estão apresentados também nesta mesma tabela. Foi realizada a análise de efeitos para a resposta e a possibilidade de obter um modelo estatisticamente significativo e preditivo para esta resposta.

A Tabela 6 apresenta-se a análise de variância para a umidade $\mathrm{t}_{120}$. Como o $\mathrm{F}_{\text {calc }}$ para regressão $(95,69)$ é altamente significativo e a porcentagem de variação explicada $\left(\mathrm{R}^{2}\right)$ pelo modelo foi considerada boa, $95 \%$, podendo-se concluir que o modelo se ajustou bem aos dados experimentais. $\mathrm{O}$ valor de $\mathrm{F}$ obtido pelo modelo foi 14,48 vezes maior que o $\mathrm{F}$ tabelado a nível de confiança de 95\%. De acordo com Rodrigues \& Iemma (2009), o modelo pode ser considerado estatisticamente significativo 
Tabela 5. Resultados do planejamento fatorial $2^{2}-$ Matriz das variáveis independentes e a variável resposta para os ensaios com casca de abacaxi enriquecida

\begin{tabular}{cccc}
\hline Ensaio & $\begin{array}{c}\mathbf{T} \\
\left({ }^{(} \mathbf{C}\right)\end{array}$ & $\begin{array}{c}\text { Var } \\
\left(\mathbf{m ~ s}^{-1}\right)\end{array}$ & $\begin{array}{c}\mathbf{X}_{(1120)} \\
(\%)\end{array}$ \\
1 & 40 & 0,8 & 9,4 \\
2 & 60 & 0,8 & 6,4 \\
3 & 40 & 1,8 & 9,3 \\
4 & 60 & 1,8 & 6,2 \\
5 & 50 & 1,3 & 8,0 \\
6 & 50 & 1,3 & 8,5 \\
7 & 50 & 1,3 & 8,3 \\
\hline
\end{tabular}

Tabela 6. Análise de variância - Umidade $\left(\mathrm{t}_{120}\right)$

\begin{tabular}{ccccc}
\hline Parâmetros & $\begin{array}{c}\text { \% variação } \\
\text { explicada } \\
\left(\mathbf{R}^{2}\right)\end{array}$ & $\mathbf{F}_{\text {Calculado }}$ & $\mathbf{F}_{\text {tabelado 1;5;0,05 }}$ & p-valor \\
& 95,03 & 95,69 & 6,61 & 0,000141 \\
\hline
\end{tabular}

quando o valor de $\mathrm{F}$ calculado for superior a duas vezes o valor de $\mathrm{F}$ tabelado, conforme ocorreu neste processo, em que o valor de $\mathrm{F}_{\text {calc }} / \mathrm{F}_{\text {tab }}$ para regressão, foi 14,48 e o valor de $\mathrm{F}_{\text {calc }} \mathrm{F}_{\text {tab }}{ }^{-1}$ para a falta de ajuste, foi 0,10 , indicando um modelo estatisticamente significativo e altamente preditivo.

Observando a superfície de resposta apresentada na Figura 4 concluiu-se que a temperatura apresentou um efeito de sinal negativo sobre a umidade $\left(\mathrm{t}_{120}\right)$ ou seja, diminuiu com o aumento da temperatura de secagem, como era esperado.

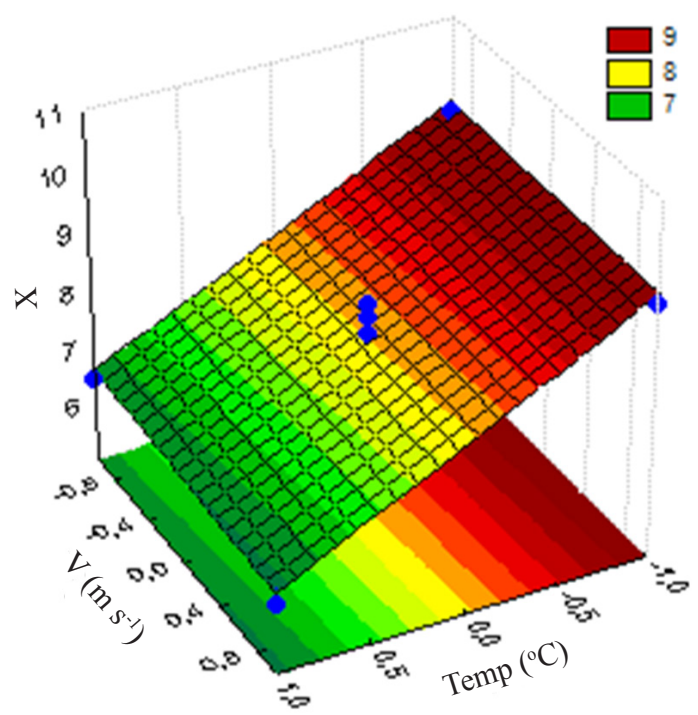

Figura 4. Superfície de resposta da umidade final do enriquecido (casca) - X em função de temperatura (Temp) e velocidade do vento (V)

Para conseguir menor teor de umidade final no resíduo enriquecido, deve-se operar a secagem com temperatura fixa em $40{ }^{\circ} \mathrm{C}$ (nível +1 ) para velocidade do ar em qualquer nível (Figura 4) obtendo-se umidade final abaixo de $7 \%$, aproximadamente $6 \%$.

Waughon \& Pena (2007) verificaram, estudando a secagem em camada delgada do resíduo fibroso de abacaxi utilizando planejamento fatorial completo com as variáveis de entrada temperatura $\left(50,60\right.$ e $\left.70^{\circ} \mathrm{C}\right)$ e velocidade de circulação do ar $\left(2,0 ; 2,5\right.$ e $\left.3,0 \mathrm{~m} \mathrm{~s}^{-1}\right)$ através da análise de variância, que a temperatura foi a variável de entrada que apresentou maior influência sobre as variáveis de resposta analisadas, similar ao que ocorreu neste trabalho.

\section{Conclusões}

1. O resíduo enriquecido estudado apresenta elevado teor de matéria seca. $\mathrm{O}$ enriquecimento proteico de casca de abacaxi mostrou-se eficiente aumentando seu teor de proteína em $165,57 \%$ em relação ao resíduo in natura.

2. Verifica-se que o modelo de Page apresenta melhor ajuste aos dados experimentais de secagem.

3. A cinética de secagem das cascas de abacaxi enriquecida mostra que a temperatura é a variável de maior influência no processo.

4. A técnica de planejamento experimental é uma ferramenta adequada para predizer resultados confiáveis durante o enriquecimento proteico e a secagem de casca de abacaxi.

\section{Literatura Citada}

Akpinar, E. K.; Bicer, Y.; Yildiz, C. Thin layer drying of red pepper. Journal of Food Engineering, v.59, p.99-104, 2003.

AOAC - Association of Official Analytical Chemists. Official methods of analysis of AOAC international. 16.ed. Maryland: AOAC, 1997. 1141p.

Azoubel, P. M.; Baima, M. do A. M.; Amorim, M. da R.; Oliveira, S. S. B. Effect of ultrasound on banana cv Pacovan drying kinetics. Journal of Food Engineering, v.97, p.194198, 2010.

Babalis, S. J.; Papanicolaou, E.; Kiriakis, N.; Belessiotis, V. G. Evaluations of thin-layer drying models for describing drying kinetics of figs (Ficus carica). Journal of Food Engineering, v.75, p.205-214, 2006.

Brasil. Instituto Adolfo Lutz. Normas analíticas do Instituto Adolfo Lutz - Métodos químicos e físicos para análise de alimentos. 2.ed. São Paulo: Brasília: Ministério da Saúde, Agência Nacional de Vigilância Sanitária, v.1, 2005. 1018p.

Brito Neto, J. F.; Pereira, W. E; Sá Sobrinho, R. G.; Barbosa; J. A.; Santos, D. P. Aspectos produtivos da abacaxicultura familiar e comercial no estado da Paraíba. Revista Caatinga, v.21, p.43-50, 2008.

Campos, A. R. N.; Santana, R. A. C de.; Dantas, J. P.; Oliveira, L. de S. C.; Silva, F. L. H. da. Enriquecimento protéico do bagaço do pendúnculo de caju por cultivo semi-sólido. Revista de Biologia e Ciências da Terra, v.5, p.1-10, 2005.

Carlesso, V. O.; Berbert, P.; A, Silva, R. F.; Detmann, E. Secagem de sementes de maracujá em camada delgada. Revista Brasileira de Fruticultura, v.27, p.444-448, 2005.

Correia, M. X de C.; Costa, R. G.; Silva, J. H. Vilar da.; Carvalho, F. F. R. de.; Medeiros, A. N. de. Utilização de resíduo agroindustrial de abacaxi desidratado em dietas para caprinos em crescimento: Digestibilidade e desempenho. Revista Brasileira de Zootecnia, v.35, p.1822-1828, 2006.

Costa, J. M. C. da.; Felipe, E. M. de F.; Maia, G. A.; Brasil, I. M. Hernandez, F. F. H. Comparação dos parâmetros físico-químicos e químicos de pós alimentícios obtidos de resíduos de abacaxi. Revista Ciência Agronômica, v.38, p.228-232, 2007. 
Della, V. P.; Kühn, I.; Hotza, D. Reciclagem de resíduos agroindustriais: Cinza de casca de arroz como fonte alternativa de sílica. Cerâmica Industrial, v.10, p.904-916, 2005.

Doymaz; I.; Pala, M. The thin-layer drying characteristics of corn. Journal of Food Engineering, v.60, p.125-130, 2003.

Figueredo, M. P. Nutrição de bovinos leiteiros e doenças metabólicas. Bahia Agrícola, v.1, p.51-61, 1996.

Fiorentin, L. D.; Menon, B. T.; Alves, J. A.; Barros, S. T. D. de.; Pereira, N. C.; Módenes, A. N. Determinação da cinética e das isotermas de secagem do bagaço da laranja. Acta Scientiarum Technology, v.32, p.147-152, 2010.

Gouveia, J. P. G. de.; Moura, R. S. F. de; Almeida, F. de A. C.; Oliveira, A. M. de V.; Silva, M. M. da. Avaliação da cinética de secagem de caju mediante um planejamento experimental. Revista Brasileira de Engenharia Agrícola e Ambiental, v.6, p.471-474, 2002.

Le Poidevin, N.; Robinson, L.A. Métodos de diagnóstico foliares utilizados nas plantações do grupo booken na Guiana Inglesa: Amostragem de análises. Fertilité, v.21, p.3-11, 1964.

Martin, L. C. T. Bovinos - Volumosos suplementares. São Paulo: Nobel, 1997. 143p.

Miller, G. Use of dinitrosalicilic acid reagent for determination of reducing sugars. Analytical Chemistry, v.31, p.426-428, 1959.

Mohapatra, D.; Rao, P. S. A thin layer drying model of parboiled wheat. Journal of Food Engineering, v.66, p.513-518, 2005.

NRC - National Research Council. Nutrient requirements of horses. Committee on Animal Nutrition, 5.ed., 1989.

Oliveira, M. M. Enriquecimento nutricional por bioconversão de resíduos agroindustriais para utilização na alimentação animal. Campina Grande: UFCG, 2007. 147p. Tese Doutorado

Pelizer, L. H.; Pontieri, M. H.; Moraes, I. O. Utilização de resíduos agro-industriais em processos biotecnológicos como perspectiva de redução do impacto ambiental. Journal of Technology Management \& Innovation, v.2, p.118-127, 2007.
Resende, O.; Corrêa, P. C.; Goneli, A. L. D.; Botelho, F. M.; Rodrigues, S. Modelagem matemática do processo de secagem de duas variedades de feijão (Phaseolus vulgaris L.). Revista Brasileira de Produtos Agroindustriais, v.10, p.17-26, 2008.

Ribas, A. I.; Cánovas, G. V. B.; Garza, S. G.; Añó, V. G. Métodos experimentales en la ingeniería alimentaria. Zaragoza: Acribia, 2000. 292p.

Ribeiro, M. E. R.; Rodrigues, R. C.; Costa, N. L. Utilização de resíduos da agroindústria na alimentação animal: In: Reunião Anual da Sociedade Brasileira de Zootecnia, 30. Rio de Janeiro. Anais... Rio de Janeiro: SBZ, 1993. $509 \mathrm{p}$.

Rodrigues, M. I.; Iemma A. F. Planejamento de experimentos e otimização de processos. Campinas: Casa do Pão Editora, 2.ed., 2009. 325p.

Silva, A. J. Análise de alimentos - Métodos químicos e biológicos. Viçosa, MG Imprensa Universitária, UFV. 166p. 1998.

Sousa, M. B. de.; Pedroza, J. P.; Beltrão, N. E. de M.; Severino, L. S.; Dantas, F. P. Cinética de secagem do farelo de mamona. Revista Brasileira de Produtos Agroindustriais, v.8, p.135-146, 2006.

Souto, R. F.; Durigan, J. F.; Souza, B. S. de; Donadon J.; Menegucci J. L. P. Conservação pós-colheita de abacaxi 'Pérola' colhido no estádio de maturação "pintado" associando-se refrigeração e atmosfera modificada. Revista Brasileira de Fruticultura, v.26, p.24-28, 2004.

Vilela, C. A. A.; Artur, P. O. Secagem do açafrão (Curcuma longa L.) em diferentes cortes geométricos. Ciência e Tecnologia de Alimentos, v.28, p.387-394, 2008.

Waughon, T. G.; Pena, R. S. Avaliação higroscópica da fibra residual do abacaxi. Revista Brasileira de Tecnologia Agroindustrial, v.1, p.83-92, 2007. 